\title{
Association of depressive symptoms with dietary habits among Japanese physicians
}

\author{
Koji Wada $^{1^{*}}$, Yukiko Suehiro ${ }^{2}$, Hiroshi Ohta ${ }^{1}$, Toru Yoshikawa $^{3}$, Takashi Hosaka $^{4}$ \\ ${ }^{1}$ Kitasato University School of Medicine, Kanagawa, Japan; ${ }^{*}$ Corresponding Author: kwada-sgy@umin.ac.jp \\ ${ }^{2}$ University of Occupational and Environmental Health, Kitakyushu, Japan \\ ${ }^{3}$ Institute for Science of Labour, Kawasaki, Japan \\ ${ }^{4}$ St. Luke's International Hospital, Tokyo, Japan
}

Received 20 June 2012; revised 22 July 2012; accepted 5 August 2012

\section{ABSTRACT}

The aim of this study was to determine the association of depressive symptoms with dietary habits among physicians working in hospitals in Japan. We mailed an anonymous questionnaire with the Quick Inventory of Depressive Symptomatology and items about dietary habits to 10,000 randomly selected physicians. Logistic regression analysis was used to explore the association of depressive symptoms with dietary habits. The participants comprised 3862 physicians (3025 men and 837 women). Among the respondents, $252(8.3 \%)$ men and $88(10.5 \%)$ women were determined to be in depressive symptoms. For men, "never eat a balanced diet" (Odds ratio; 95\% confidence interval, $2.54 ; 1.70$ 3.80), and for women "eat quite small meals" (3.81; 1.14 - 13.1), "always eat until full" (4.40; 1.48 - 13.1), and "never eat a balanced diet" (3.05; 1.49 - 6.23) were associated with depressive symptoms. For men, "do not eat too much" (0.57; $0.39-0.83$ ) was associated with prevention of depression. Physicians should recognize the important role of healthy dietary habits in good mental health. Hospitals should intervene to help physicians address depressive symptoms and dietary habits.

Keywords: Physicians; Depression; Nutrition; Food Habits

\section{INTRODUCTION}

Physicians tend to have poor lifestyles because of long working hours and heavy workloads [1]. Poor lifestyles have been associated with mental disorders among the general population [2], and physicians are no exception. Poor dietary habits have also been associated with de- pressive symptoms [3]. However, interventions to improve dietary habits may help prevent mental disorders. For example, among emergency rescue workers, omega3 polyunsaturated fatty acid supplements attenuated posttraumatic distress [4]. The aim of this study was to determine the association of depressive symptoms with dietary habits among physicians working in hospitals in Japan.

\section{MATERIALS AND METHODS}

This study was conducted by the Japan Medical Association's committee for physicians' health. We mailed an anonymous self-administered questionnaire to 10,000 (8000 men and 2000 women) randomly selected physicians working at hospitals who were members of the Japan Medical Association ( $\mathrm{n}=75,951$ physicians). We did not send any reminders to the population in this study.

The question regarding meal size was "How much do you usually eat at meal for a previous year?” The answer choices were "Quite small meals", "Always moderately-sized meals", "Do not eat too much", "Eat rather too much", and "Always eat until full". Concerning dietary balance, the question was "How often do you eat balanced diet consisting of a principal food (e.g., rice, bread, etc.), main dish (e.g., meat, fish, egg, and soybeans, etc.) and side dishes (e.g., vegetables, seaweed, etc.)?" The answer choices were "Every meal", "Two meals a day", "One meal a day", and "Never".

Physicians with depressive symptoms were identified by a score of 11 or more on the Japanese version of the Quick Inventory Depressive Scale-Self Report (QIDSSR) [5]. The QIDS-SR comprises 16 questions with a 4point Likert scale as a measure of the severity of depressive symptoms. Total scores for depression on the QIDS-SR range from 0 to 27 ( 0 to $5=$ none, 6 to $10=$ mild, 11 to 15 = moderate, 16 to $20=$ severe, and 21 to 27 = very severe)

Logistic regression analysis was used to explore the 
association of depressive symptoms with dietary habits. We adjusted for age, working factors which were associated with depressive symptoms in previous studies [1,6] such as the number of overnight works, on-calls, and off-duties, average hour of sleeping not doing overnight work, and unreasonable violence at work. We also adjusted for lifestyle factors such as smoking, alcohol intake, and regular exercise. A two-tailed value of $\mathrm{p}<0.05$ was considered to be significant. Odds ratios (ORs) $>1.0$ indicated increased risk and Ors $<1.0$ indicated protecttive factors for depressive symptoms. All analyses were performed using the Statistical Package for the Social Sciences (SPSS) for Windows, ver. 15.0.

The Human Research Committee at the Institute for Science of Labour approved the procedure before the study commenced.

\section{RESULTS}

Of the respondents, 31 did not receive the questionnaire. An additional 176 did not qualify for the study because they no longer worked at hospitals, were on maternity leave, or had retired. A total of 3862 individuals (3025 men and 837 women) completed the questionnaire, with an adjusted response rate of $40.5 \%$.

Table 1 shows the characteristics of participants. The age of male and female respondents predominantly ranged from 40 to 59 years and 30 to 49 years, respectively. A total of $8.2 \%$ of men and $10.4 \%$ of women were identified as having depressive symptoms. With regard to meal size, more than half of respondents did not eat too much, while a small percentage ate quite small meals or always ate until full. More than $10 \%$ of respondents reported never eating balanced meals.

Table 2 shows the results of the univariate and logistic regression analyses of the association of depressive symptoms with dietary habits. In the adjusted model, for both men and women, depressive symptoms were significantly associated with never eating balanced meals (odds ratio, 2.54; 95\% confidence interval, 1.70 - 3.80 for men and 3.05; 1.49 - 6.23 for women). The dietary habit of not eating too much $(0.57 ; 0.39$ - 0.83) was negatively associated with depressive symptoms for men. The dietary habits of eating quite small meals (3.81; 1.14 - 13.1) and always eating until full (4.40; 1.48 - 13.1) were associated with depressive symptoms for women.

\section{DISCUSSION}

Poor dietary habits were associated with depressive symptoms among physicians working in hospitals in Japan. Interestingly, healthy dietary habits such as not eating too much at meal were a preventive factor for depression in men. There were some physicians who were not able to eat a balanced diet daily.
Table 1. Characteristics of participants by gender.

\begin{tabular}{lcccc}
\hline & \multicolumn{2}{c}{ Men } & \multicolumn{2}{c}{ Women } \\
\cline { 2 - 5 } & $\mathrm{n}=3025$ & $(\%)$ & $\mathrm{n}=837$ & $(\%)$ \\
\hline Age (years) & 38 & $(1.3)$ & 51 & $(6.1)$ \\
$24-29$ & 417 & $(13.7)$ & 300 & $(35.8)$ \\
$30-39$ & 876 & $(29.0)$ & 270 & $(32.3)$ \\
$40-49$ & 967 & $(32.0)$ & 141 & $(16.8)$ \\
$50-59$ & 464 & $(15.3)$ & 55 & $(6.6)$ \\
$60-69$ & 263 & $(8.7)$ & 20 & $(2.4)$ \\
70 or older &
\end{tabular}

The score of quick inventory depressive scale

$\begin{array}{lcccc}0-10 & 2773 & (91.7) & 749 & (89.5) \\ 11-15 & 199 & (6.6) & 66 & (7.9) \\ 16-20 & 41 & (1.4) & 18 & (2.2) \\ 21-27 & 12 & (0.4) & 4 & (0.5) \\ \text { Usual meal size } & & & & \\ \text { Quite small } & 124 & (4.1) & 20 & (2.4) \\ \text { Always moderate } & 521 & (17.2) & 124 & (14.8) \\ \text { Do not eat too much } & 1667 & (55.2) & 512 & (61.3) \\ \text { Eat rather too much } & 635 & (21.0) & 152 & (18.1) \\ \text { Always eat until full } & 78 & (2.5) & 29 & (3.4)\end{array}$

Frequency of balanced diet consisting of a principal food, a main dish, and side dishes

\begin{tabular}{lcccc} 
Every meal & 855 & $(28.3)$ & 167 & $(20.0)$ \\
Two meals a day & 1097 & $(36.2)$ & 321 & $(38.4)$ \\
One meal a day & 763 & $(25.2)$ & 254 & $(30.3)$ \\
Never & 310 & $(10.2)$ & 95 & (11.3) \\
\hline
\end{tabular}

Physicians at hospitals tend to eat meals irregularly because of their duties. However, not eating too much at meal, thus maintaining a healthy lifestyle through exercising self-control and willpower, could prevent depresssion in men. In women, an association between eating too little/too much and depression was observed, suggesting subclinical depressive symptoms such as appetite loss and binge eating. Women may react more strongly to perceived stress, and depressive symptoms may be reflected in their food intake [7].

Dietary patterns characterized by high intake of vegetables, fruits and fish were associated with lower incidence of depression $[3,8,9]$. A lack of specific nutrients such as folic acid [10] and vitamin B [11] reportedly increase the risk of depression. In this study, in both male and female physicians, never eating balanced meals was significantly associated with depressive symptoms. As an alternative explanation, those physicians seem to have 
Table 2. Association of depressive symptoms with dietary habits among physicians working in hospitals in Japan.

\begin{tabular}{|c|c|c|c|c|}
\hline & \multicolumn{2}{|c|}{ Men } & \multicolumn{2}{|c|}{ Women } \\
\hline & $\begin{array}{c}\text { Univariate } \\
\text { OR (95\% CI) }\end{array}$ & $\begin{array}{l}\text { Multivariate } \\
\text { OR (95\% CI) }\end{array}$ & $\begin{array}{c}\text { Univariate } \\
\text { OR (95\% CI) }\end{array}$ & $\begin{array}{l}\text { Multivariate } \\
\text { OR (95\% CI) }\end{array}$ \\
\hline \multicolumn{5}{|l|}{ Usual meal size } \\
\hline Quite small & $1.85(1.06-3.23)$ & $1.38(0.74-2.61)$ & $6.35(2.07-19.5)$ & $3.81(1.14-13.1)^{*}$ \\
\hline Always moderately & 1.00 & 1.00 & 1.00 & 1.00 \\
\hline Do not eat too much & $0.64(0.45-0.90)$ & $0.57(0.39-0.83)^{*}$ & $0.95(0.46-1.96)$ & $0.74(0.34-1.62)$ \\
\hline Eat rather too much & $1.09(0.74-1.61)$ & $0.78(0.51-1.20)$ & $2.12(0.97-4.64)$ & $1.64(0.70-3.83)$ \\
\hline Always eat until full & $1.78(0.90-3.52)$ & $0.72(0.31-1.69)$ & $6.56(2.39-18.0)$ & $4.40(1.48-13.1)^{*}$ \\
\hline \multicolumn{5}{|c|}{ Frequency of balanced diet consisting of a principal food, a main dish, and side dishes } \\
\hline Every meal & $0.70(0.48-1.04)$ & $0.67(0.45-1.02)$ & $0.83(0.40-1.74)$ & $0.95(0.43-2.10)$ \\
\hline Two meals a day & 1.00 & 1.00 & 1.00 & 1.00 \\
\hline One meal a day & $1.44(1.03-2.02)$ & 1.35 (0.95 - 1.93) & $1.53(0.87-2.68)$ & $1.48(0.80-2.74)$ \\
\hline Never & $3.27(2.27-4.27)$ & $2.54(1.70-3.80)^{*}$ & $3.81(2.04-7.10)$ & $3.05(1.49-6.23)^{*}$ \\
\hline
\end{tabular}

no time for socializing activities, including having dinner at home or sharing lunchtime with colleagues, which may contribute to coping with stressful events.

A limitation of this study should be noted. Because this was a cross-sectional design, we could not confirm causal relationships. However, it could be helpful to identify physicians who need intervention for depressive symptoms and poor dietary habits in an effort to support their mental health. In addition, there could be other confounding factors which were not identified and we do not have any further information about non-respondents. In the end, our response rate was not optimal probably due to lack of repeated reminders.

In conclusion, physicians should recognize the importance of healthy dietary habits in maintaining mental health. Since physicians need to stay at the hospital for long hours, sometimes overnight, hospitals should support their good dietary habits.

\section{ACKNOWLEDGEMENTS}

We thank all the physicians who participated in this research. We also thank Prof. Takahisa Goto, Dr. Aizan Hirai, Dr. Eisuke Matsushima, Dr. Yoshifumi Nakashima, Dr. Rie Akaho, and Dr. Michiko Kido who are members of the working committee on physicians' health, Japan Medical Association, and Dr. Satoshi Imamura, executive board member of the Japan Medical Association.

\section{REFERENCES}

[1] Wada, K., Yoshikawa, T., Goto, T., Hirai, A., Matsushima E., et al. (2010) National survey of the association of depressive symptoms with the number of off duty and on-call, and sleep hours among physicians working in Japanese hospitals: A cross sectional study. BMC Public Health, 10, 127. doi:10.1186/1471-2458-10-127

[2] Wada, K., Satoh, T., Tsunoda, M. and Aizawa, Y. (2006) Associations of health behaviors on depressive symptoms among employed men in Japan. Industrial Health, 44, 486-492. doi:10.2486/indhealth.44.486

[3] Nanri, A., Kimura, Y., Matsushita, Y., Ohta, M. and Sato, M., et al. (2010) Dietary patterns and depressive symptoms among Japanese men and women. European Journal of Clinical Nutrition, 64, 832-839. doi:10.1038/ejcn.2010.86

[4] Matsuoka, Y., Nishi, D., Nakaya, N., Sone, T., Hamazaki, K., et al. (2011) Attenuating posttraumatic distress with omega-3 polyunsaturated fatty acids among disaster medical assistance team members after the Great East Japan Earthquake: The APOP randomized controlled trial. BMC Psychiatry, 11, 132. doi:10.1186/1471-244X-11-132

[5] Rush, A.J., Trivedi, M.H., Ibrahim, H.M., Carmody, T.J., Arnow, B., et al. (2003) The 16-Item Quick Inventory of Depressive Symptomatology (QIDS), clinician rating (QIDS-C), and self-report (QIDS-SR): A psychometric evaluation in patients with chronic major depression. Biological Psychiatry, 54, 573-583. doi:10.1016/S0006-3223(02)01866-8

[6] Wada, K., Yoshikawa, T., Goto, T., Hirai, A., Matsushima, E., et al. (2011) Association of depression and suicidal ideation with unreasonable patient demands and complaints among Japanese physicians: A national crosssectional survey. International Journal of Behavioral Medicine, 18, 384-390. doi:10.1007/s12529-010-9132-7

[7] Mikolajczyk, R.T., El Ansari, W. and Maxwell, A.E. (2009) 
Food consumption frequency and perceived stress and depressive symptoms among students in three European countries. Nutrition Journal, 8, 31. doi:10.1186/1475-2891-8-31

[8] Akbaraly, T.N., Brunner, E.J., Ferrie, J.E., Marmot, M.G., Kivimaki, M., et al. (2009) Dietary pattern and depressive symptoms in middle age. British Journal of Psychiatry, 195, 408-413. doi:10.1192/bjp.bp.108.058925

[9] Sanchez-Villegas, A., Delgado-Rodriguez, M., Alonso, A., Schlatter, J., Lahortiga, F., et al. (2009) Association of the Mediterranean dietary pattern with the incidence of depression: The Seguimiento Universidad de Navarra/ University of Navarra follow-up (SUN) cohort. Archives of General Psychiatry, 66, 1090-1098. doi:10.1001/archgenpsychiatry.2009.129

[10] Murakami, K., Mizoue, T., Sasaki, S., Ohta, M., Sato, M., et al. (2008) Dietary intake of folate, other B vitamins, and omega-3 polyunsaturated fatty acids in relation to depressive symptoms in Japanese adults. Nutrition, 24, 140-147. doi:10.1016/j.nut.2007.10.013

[11] Skarupski, K.A., Tangney, C., Li, H., Ouyang, B., Evans D.A., et al. (2010) Longitudinal association of vitamin B-6, folate, and vitamin B-12 with depressive symptoms among older adults over time. American Journal of Clinical Nutrition, 92, 330-335. doi:10.3945/ajcn.2010.29413 